\title{
Prognostic value of impulsivity in treatment outcomes in patients with alcohol use and/or cocaine use disorder in a rehabilitation programme
}

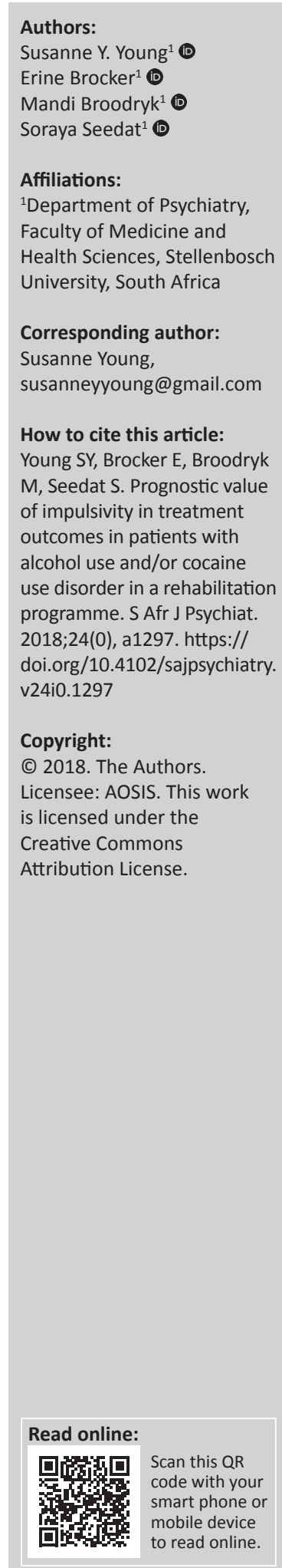

Introduction: Impulsivity is linked to factors that are negatively correlated with drug and alcohol use. Individuals with substance use disorder (SUD) often suffer from cognitive deficits and, additionally, have high levels of impulsivity. Studies show that cognitive deficits are associated with lower self-efficacy (SE), and the latter is considered an important indicator of SUD management and treatment outcomes. The relationship between impulsivity and SE, however, remains unclear. This prospective study examined impulsivity as a prognostic indicator for SE in SUD populations admitted for inpatient treatment.

Methods: Fifty individuals, aged 18-61, with either a cocaine use and/or alcohol use disorder diagnosis were examined within 72 hours of (1) the start and (2) completion of treatment.

Results: Impulsivity was a significant predictor of SE. Duration of abstinence (in days), estimated intelligence, global assessment of functioning (GAF) and patient age explained $16 \%$ of the variance in the change in SE at discharge. After including impulsivity in the regression model, the total variance explained by the model was $28 \%(F[5.505]=3.47, p=0.01)$. Impulsivity explained an additional $12 \%$ of the variance after controlling for the above variables $\left(R^{2}\right.$ change $=0.12, F$ change $\left.[4.45]=7.206, p=0.01\right)$.

Conclusion: Impulsivity is a significant predictor of SE following an 8-week impatient treatment programme for individuals diagnosed with SUD. To our knowledge, this is the first study to demonstrate that impulsivity holds prognostic value in respect of the change in SE after inpatient treatment of individuals with SUD. Based on our findings, replication studies are warranted.

Note: The authors would like to acknowledge the University of Stellenbosch, South Africa for their support of this project; the University of Amsterdam and Prof. Anneke Goudriaan for their support and guidance; the UCLA/South Africa Chronic Mental Disorders Research Training Programme for their support and guidance; Momentum GGZ, Veldhoven, the Netherlands, and Momentum Mental Healthcare and Addiction Care, South Africa, for their support to this project. They would also like to acknowledge the Hendrik Vrouwes Foundation, Nedbank, South Africa; The National Research Foundation South Africa; and the South African Research Chairs Initiative of the Department of Science and Technology for their financial support to this project. In addition, they would like to thank Dr Lize Weich for her support to this project, and Justine Blampain, Merel van Gelder, Anouk Albien, and Bodine van Styrum for their research assistance.

A selected abstract from papers presented at the 19th National Congress of the South African Society of Psychiatrists in 'Professional Psychiatric Practice: Medical, Socio-Economic \& Cultural Perspectives', 21-24 September 2018, at the CSIR, Pretoria, South Africa. The congress is hosted by South African Society of Psychiatrists (SASOP). 